\title{
ON INVERSES OF PRODUCTS OF IDEMPOTENTS \\ IN REGULAR SEMIGROUPS
}

D. G. FITZ-GERALD

(Received 12 January 1970)

Communicated by G. B. Preston

Let $E$ be the set of idempotents of a regular semigroup; we prove that $V\left(E^{n}\right)=E^{n+1}$ (see below for the meaning of this notation). This generalizes a result of Miller and Clifford ([3], theorem 4, quoted as exercise 3(b), p. 61, of Clifford and Preston [1]) and the converse, proved by Howie and Lallement ([2], lemma 1.1), which together establish the case $n=1$. As a corollary, we deduce that the subsemigroup generated by the idempotents of a regular semigroup is itself regular.

Let $N$ denote the set of natural numbers; let $n \in N$ and $S$ be any semigroup. We denote by $E$ the set of idempotents of $S$ and by $E^{n}$ the set of all products of $n$ idempotents of $S$. Further, if $E$ is not empty, let $\langle E\rangle$ denote the subsemigroup of $S$ generated by $E$; then $\langle E\rangle=\bigcup_{i \in N} E^{i}$.

For any $x \in S$, we set

$$
V(x)=\{y \in S: x y x=x \text { and } y x y=y\},
$$

the set of all inverses of $x$; and for any subset $X$ of $S$ we set $V(X)=\bigcup_{x \in X} V(x)$. For $m \in N$, we define $V^{m}(X)$ inductively, thus: $V^{m+1}(X)=V\left(V^{m}(X)\right)$.

Lemma 1. Let $y \in S$. Then $y \in y E^{n} y$ implies $y \in E^{n+1}$.

Proof. Suppose $y=y x y$ for some $x \in E^{n}$, say $x=e_{1} \cdots e_{n}$, where $e_{i} \in E$ for $i=1, \cdots, n$.

For $i=1, \cdots, n$, set $t_{i}=e_{1} \cdots e_{i}$ and $u_{i}=e_{i} \cdots e_{n}$, so that $t_{i} u_{i}=x$. If $n \geqq 2$ set, for $j=2, \cdots, n, f_{j}=u_{j} y t_{j-1}$, so that

that is, $f_{j} \in E$. But

$$
\begin{aligned}
f_{j}^{2}=u_{j} y t_{j-1} u_{j} y t_{j-1} & =u_{j} y x y t_{j-1} \\
& =u_{j} y t_{j-1} \\
& =f_{j},
\end{aligned}
$$

$$
\begin{aligned}
y=y x y & =y(x y)^{n} \\
& =y \cdot t_{n} u_{n} y \cdot t_{n-1} u_{n-1} y \cdots t_{1} u_{1} y \\
& =y t_{n} \cdot u_{n} y t_{n-1} \cdots u_{2} y t_{1} \cdot u_{1} y \\
& =y x \cdot f_{n} \cdots f_{2} \cdot x y . \\
& 335
\end{aligned}
$$


(In the above, an undefined symbol is to be understood as the empty symbol.) Since $x y, y x \in E$, it follows that $y \in E^{n+1}$.

Corollary. $V\left(E^{n}\right) \subseteq E^{n+1}$.

LEMMA 2. Let $S$ be regular. Then $E^{n+1} \subseteq V\left(E^{n}\right)$.

Proof. Let $x=e_{1} \cdots e_{n+1}$, where $e_{i} \in E$ for $i=1, \cdots, n+1$. Since $S$ is regular, there exists some $y \in S$ such that $x y x=x$ and $y x y=y$.

For $i=1, \cdots, n+1$, set $t_{i}=e_{1} \cdots e_{i}$ and $u_{i}=e_{i} \cdots e_{n+1}$, so that $t_{i} u_{i}=x$. Further, for $j=1, \cdots, n$, set $f_{j}=u_{j+1} y t_{j}$, so that

$$
f_{j}^{2}=u_{j+1} y t_{j} u_{j+1} y t_{j}=f_{j} .
$$

Then $z=f_{n} \cdots f_{1} \in E^{n}$. But

$$
\begin{aligned}
x & =x y x=x(y x)^{n} \\
& =x e_{n+1} \cdot y t_{n} u_{n} \cdots y t_{1} u_{1} \\
& =x \cdot u_{n+1} y t_{n} \cdots u_{2} y t_{1} \cdot u_{1} \\
& =x f_{n} \cdots f_{1} x \\
& =x z x
\end{aligned}
$$

and

$$
\begin{aligned}
z x z & =f_{n} \cdots f_{1} x f_{n} \cdots f_{1} \\
& =f_{n} \cdots f_{2} \cdot u_{2} y t_{1} \cdot x \cdot u_{n+1} y t_{n} \cdots u_{2} y t_{1} \\
& =f_{n} \cdots f_{2} \cdot u_{2} y \cdot t_{1} x u_{n+1} \cdot y t_{n} u_{n} \cdots y t_{2} u_{2} \cdot y t_{1} \\
& =f_{n} \cdots f_{2} \cdot u_{2} y \cdot e_{1} x e_{n+1} \cdot(y x)^{n-1} y t_{1} \\
& =f_{n} \cdots f_{2} u_{2} y x \cdot y x y t_{1} \\
& =f_{n} \cdots f_{2} u_{2} y t_{1} \\
& =z .
\end{aligned}
$$

Thus $x \in V(z) \subseteq V\left(E^{n}\right)$, and the lemma is proved.

Lemmas 1 and 2 together establish the

THEOREM. Let $S$ be regular. Then $V\left(E^{n}\right)=E^{n+1}$.

COROLlary. Let $S$ be regular. Then $\langle E\rangle$ is regular.

REMARK. Moreover, $\langle E\rangle$ is then a complete regular subsemigroup of $S$ in the sense that each inverse in $S$ of an element of $\langle E\rangle$ is a member of $\langle E\rangle$. Indeed,

$$
\langle E\rangle=E \cup\left(\bigcup_{m \in N} E^{m}\right)=\bigcup_{m \in N} V^{m}(E) .
$$

The author thanks his supervisor, Dr. T. E. Hall, for suggesting lemma 2. 


\section{References}

[1] A. H. Clifford and G. B. Preston, The algebraic theory of semigroups, Volume 1, (Math. Surveys, No. 7, Amer. Math. Soc., 1961).

[2] J. M. Howie and G. Lallement, 'Certain fundamental congruences on a regular semigroup', Proc. Glasgow Math. Assoc. 7 (1966), 145-159.

[3] D. D. Miller and A. H. Clifford, 'Regular $\mathscr{D}$-classes in semigroups', Trans. Amer. Math. Soc. 82 (1956), 270-280.

Department of Mathematics

Monash University, Clayton 